\title{
Helical-structure-induced softening of particle/liquid-crystal composite gels
}

\author{
Takahiro Yamamoto*, Yuki Kawata, Hideyuki Kihara and Masaru Yoshida \\ Nanosystem Research Institute, National Institute of Advanced Industrial Science and Technology, \\ Central 5, 1-1-1 Higashi, Tsukuba, Ibaraki 305-8565, JAPAN \\ * Corresponding author: e-mail: takahiro.yamamoto@aist.go.jp
}

\begin{abstract}
We investigated the effects of the introduction of helical structures into a host liquid crystal on the viscoelastic properties of particle/liquid-crystal composite gels. A composite gel using a cholesteric liquid crystal as a host matrix was found to become soft compared to that using a nematic liquid crystal. With reducing the half-pitch length of a helical structure of cholesteric liquid crystals, a storage modulus of the composite gels showed a linear decrease. On the other hand, a crossover point which is a mechanical quasi-solid-quasi-liquid transition point exhibited no significant changes when the half-pitch length was larger than the radius of a particle used in this study. However, a decrease of the crossover points was observed when the half-pitch length was shorter than the particle radius. We qualitatively discussed the origin of helical-structure-induced softening of particle/liquid-crystal composite gels based on the differences in self-organization behavior of particles in nematic and cholesteric liquid crystals.
\end{abstract}

Key words: liquid crystal, composite, gel, nematic phase, cholesteric phase

\section{INTRODUCTION}

Particle/liquid-crystal (LC) composite gels are a kind of liquid-crystalline physical gels and have recently attracted an increasing attention due to their scientific and technological interests [1-12]. In particle/LC composite gels, micro- and nano-sized particles act as a sort of gelators and form three-dimensional networks in host LCs $[4,5]$. So far, a few applications of particle/LC composite gels have been proposed, such as chemical sensors and cell culture media $[11,12]$. Recently, we reported the development of novel photo-healable materials consisting of particle/LC composite gels doped with an azobenzene derivative [9]. By means of the gel-sol transition induced by trans-cis photoisomerization of an azo-dye, we achieved light-assisted mending of surface cracks on the particle/LC composite gels. In addition, we previously investigated effects of the phase structures (nematic and smectic A phases) of host LCs on the viscoelastic properties of particle/LC composite gels [10]. By the introduction of a layer structure of molecular organization into a host LC matrix, we found that a storage modulus indicating the rigidness of the composite gels showed an increase of approximately one order of magnitude with no significant reduction of a crossover point which denotes the toughness of the composite gels. In this study, we investigated effects of the introduction of a helical structure of molecular alignment into the host matrix using cholesteric LCs on the viscoelastic properties of particle/LC composite gels.

\section{EXPERIMENTAL SECTION}

\subsection{Materials}

Fig. 1 shows the chemical structures of a nematic LC mixture (ZLI-1083) and chiral dopants (S811 and R811). Cholesteric LCs were prepared by doping ZLI-1083 with the mixtures of S811 and R811 having the different enantiomeric excess (e.e.) values. The concentrations of the mixtures of S811 and R811 in cholesteric LCs were adjusted at 5 or $15 \mathrm{wt} \%$. The $e$. e. value was calculated using a following equation (Eq. 1). The $e . e$. value = $100 \%$ means that ZLI-1083 contains only S811. A half-pitch length (pitch/2) of cholesteric LCs was measured by a conventional wedge cell method at $25^{\circ} \mathrm{C}$.

$$
\text { e. e. }(\%)=\frac{([\mathrm{S} 811]-[\mathrm{R} 811])}{([\mathrm{S} 811]+[\mathrm{R} 811])} \times 100
$$

Monodisperse polymeric particles (Micropearl, EX-0036-AC6, radius $=1.8 \mu \mathrm{m}$ ) consisting of polymerized styrene and divinylbenzene were purchased from Sekisui Chemical Co. Ltd. The particles were the same as the previous studies $[9,10]$.

\subsection{Sample preparation}

The polymeric particles were weighed and suspended in each host cholesteric LC in a glass vial. The
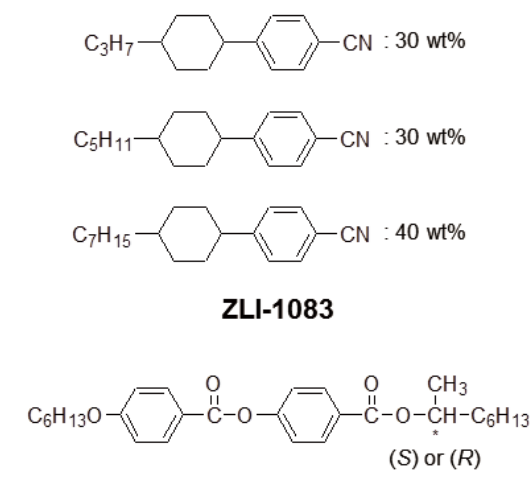

(S) form: $\mathbf{5 8 1 1}$

$(R)$ form: $\mathbf{R 8 1 1}$

Fig. 1 Chemical structures of liquid-crystalline mixture (ZLI-1083) and chiral dopants (S811 and R811) used in this study. 
concentration of the polymeric particle to cholesteric LCs was $33 \mathrm{wt} \%$. In this study, in order to allow discuss the effects of phase structures of LCs, we employed almost the same particle concentration as the previous study [10]. The suspension was heated above the clearing temperature of the host cholesteric LCs on a hot plate and vigorously mixed to ensure the homogeneous dispersion of the particles. The suspension was then cooled to $25{ }^{\circ} \mathrm{C}$ at a fixed rate of about $10{ }^{\circ} \mathrm{C} / \mathrm{min}$. At the phase-transition point from an isotropic phase to a cholesteric one, the gel formation was induced. Each sample was kept overnight at $25{ }^{\circ} \mathrm{C}$ before each experiment was performed.

\subsection{Measurements}

Viscoelastic properties of particle/LC composite gels were investigated using an Advanced Rheometric Expansion System (ARES-RFS, TA Instruments, Rheometric Scientific) with a parallel-plate type geometry (plate diameter: $25 \mathrm{~mm}$ ). Dynamic frequency and strain sweep tests were conducted at $25{ }^{\circ} \mathrm{C}$. A frequency sweep test was performed in a strain-controlled mode (strain: 0.1\%). A strain sweep test was conducted at the frequency of $1 \mathrm{~Hz}$. A gap of $0.75 \mathrm{~mm}$ was left between the plates to be filled with the composite gels.
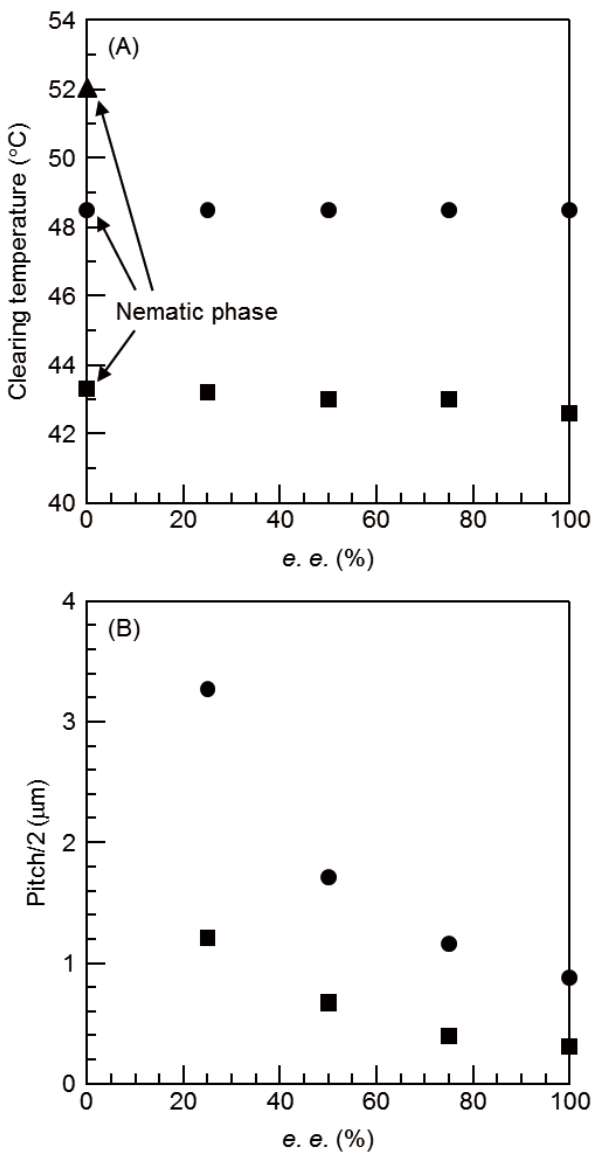

Fig. 2 Clearing temperatures (A) and half-pitch length (pitch/2) values (B) of host cholesteric LCs as a function of $e$. $e$. values. The concentrations of the mixture of the chiral dopants were $0(\boldsymbol{\Delta}), 5(\bullet)$, and $15(\bullet) \mathrm{wt} \%$.
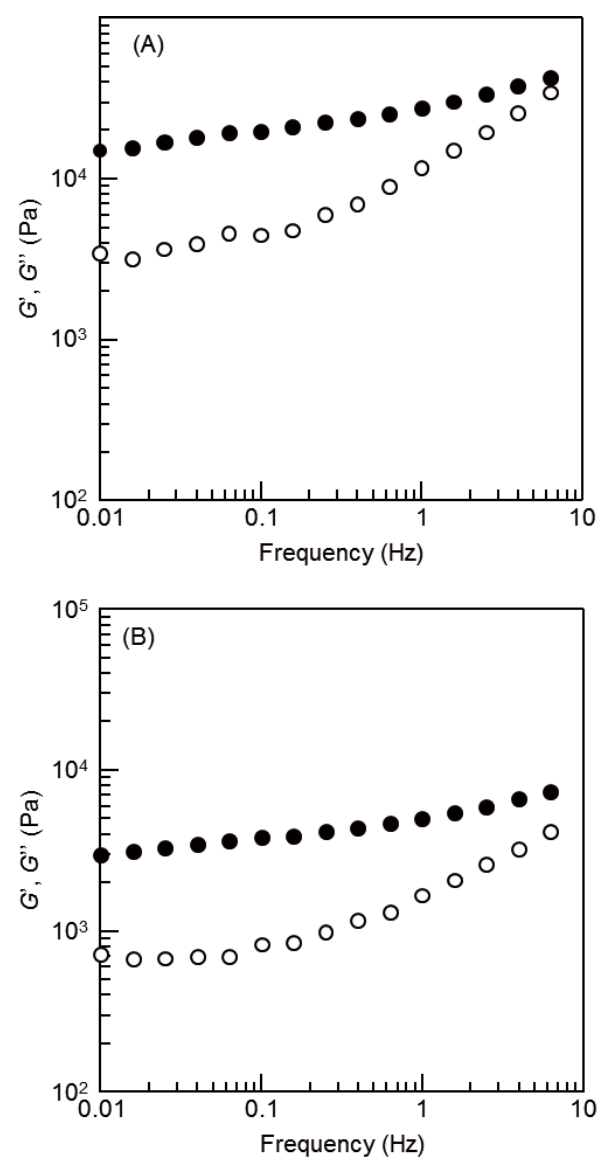

Fig. 3 Frequency dependency of storage $\left(G^{\prime}\right.$, -) and loss $\left(G^{\prime \prime}, \circ\right)$ moduli of particle/LC composite gels with different $e$. e. values: (A) $0 \%$ (nematic phase); (B) $100 \%$ (cholesteric phase, $\operatorname{pitch} / 2=0.88 \mu \mathrm{m})$. The concentration of the chiral dopants was $5 \mathrm{wt} \%$ for both composite gels. Experiments were conducted at $25^{\circ} \mathrm{C}$.

\section{RESULTS AND DISCUSSION}

3.1 Physical properties of cholesteric host LCs

Fig. 2 shows the clearing temperatures of host cholesteric LCs and pitch/2 values of helical structures as a function of $e$. $e$. values. Although, the addition of the non-liquid-crystalline chiral dopants (S811 and R811) simply induced a reduction of the clearing temperatures of ZLI-1083 from $52(\boldsymbol{\Lambda}, 0 \mathrm{wt} \%)$ to 48.5 $(\bullet, 5 \mathrm{wt} \%)$ and $43.3(\bullet, 15 \mathrm{wt} \%){ }^{\circ} \mathrm{C}$ at $e . e .=0 \%$ (nematic phase), the clearing temperature of cholesteric LCs $(e . e .>0 \%)$ did not show any significant changes even if the $e$. $e$. value changed at each dopant concentration (Fig. 2 (A)). Meanwhile, the pitch/2 values of the helical structure became shorter with increasing the $e$. $e$. value (Fig. 2 (B)). Therefore, in this study, we can discuss the effects of (i) the introduction of a helical structure and (ii) pitch lengths of a helical structure on the viscoelastic properties of the composite gels at each dopant concentration.

3.2 Effects of helical structures on the viscoelastic properties of particle/LC composite 


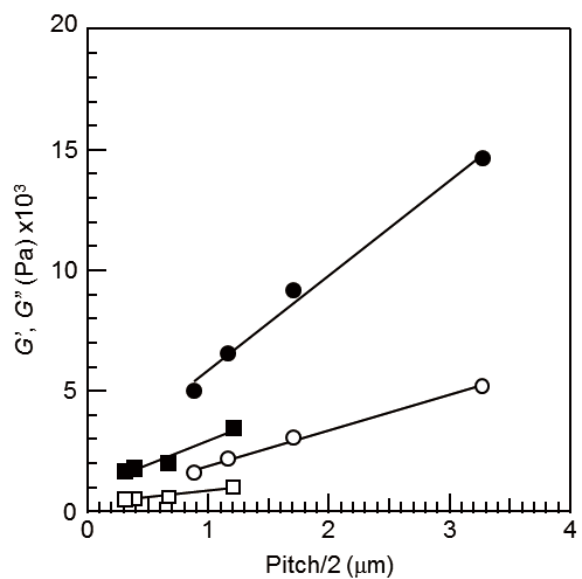

Fig. 4 Storage $\left(G^{\prime}\right.$, filled symbols) and loss $\left(G^{\prime \prime}\right.$, open symbols) moduli of particle/LC composite gels as a function of pitch/2 of cholesteric LCs. The concentrations of chiral dopants were $5(\bullet, \circ)$ and $15(\mathbf{a}, \square)$ wt $\%$. Experiments were carried out at $25^{\circ} \mathrm{C}$. gels

Fig. 3 shows the frequency dependence of viscoelastic properties (storage and loss moduli) of particle/LC composite gels (the concentration of the mixture of the dopants was $5 \mathrm{wt} \%$ and e.e. $=0$ or $100 \%$ ). In this frequency region, we confirmed that both composite gels exhibited quasi-solid nature $\left(G^{\prime}>G^{\prime \prime}\right)$ similarly to the previous reports $[9,10]$. As a representative value, at a frequency of $1 \mathrm{~Hz}$, the values of the storage modulus which indicate the rigidness of the composite gels are $2.9 \times 10^{4}$ and $0.5 \times 10^{4} \mathrm{~Pa}$ at $e . e$. $=0$ and $100 \%$, respectively. We therefore found that the storage modulus was reduced by the introduction of a helical structure into a LC matrix even though the clearing temperatures of both composite gels were almost the same as shown in Fig. 2(A) $\left(T_{\mathrm{c}}=48.5^{\circ} \mathrm{C}\right)$. As opposed to the case of the introduction of a layer structure into a host LC [10], these results clearly indicate that helical structures make the composite gels soft. Similar results were also observed in the composite gels containing the chiral dopants of $15 \mathrm{wt} \%$ (data not shown). Fig. 4 shows the effect of pitch $/ 2$ values on the storage and loss moduli of the particle/LC composite gels. In both concentrations of the chiral dopants, the composite gels became softer as the pitch/2 values became smaller. In addition, we found that both moduli of the composite gels can be continuously tuned by changing the pitch length of a helical structure in each composite gel. This would be a novel manner of the control of the viscoelastic properties of particle/LC composite gels.

Next, we investigated effects of helical structure on a crossover point at which a mechanical quasi-solid-quasi-liquid transition occurred, in other words, the crossover point indicates the toughness of the composite gels against mechanical strain. Fig. 5 shows the results of strain sweep tests of the composite gels containing the chiral dopants of $5 \mathrm{wt} \%$. The crossover point was slightly reduced from 11.9 (Fig. 5A, e. e. $=$ $0 \%$ ) to $9.1 \%$ (Fig. $5 \mathrm{~B}$, e. e. $=100 \%$ ) by the introduction of a helical structure into the host matrix. However, the reduction of the crossover point strongly depended on the pitch/2. Fig. 6 shows the effect of pitch/2 values on the crossover points of the composite gels containing the chiral dopants of 5 and $15 \mathrm{wt} \%$. When the pitch $/ 2$ value was larger than approximately $1.8 \mu \mathrm{m}$, the crossover points did not show significant changes. However, the crossover points exhibited a clear decrease when the pitch $/ 2$ values were smaller than approximately $1.8 \mu \mathrm{m}$. Interestingly, this pitch/2 value is almost the same as the radius of particles used in this study.

Self-organizing behavior of particles in nematic and cholesteric LCs was previously investigated [6]. In that study, the authors reported that particles tended to form one-dimensional chains in nematic LCs. One-dimensional chains could easily propagate to all direction in a nematic matrix. Therefore, we expect that the three-dimensional networks would be easily constructed with particles in nematic LCs. On the other hand, in cholesteric LCs, two-dimensional plates were predominantly formed with particles. The authors revealed that two-dimensional plates propagated preferentially within the same plane. In such a situation, we infer that the formation of firm three-dimensional
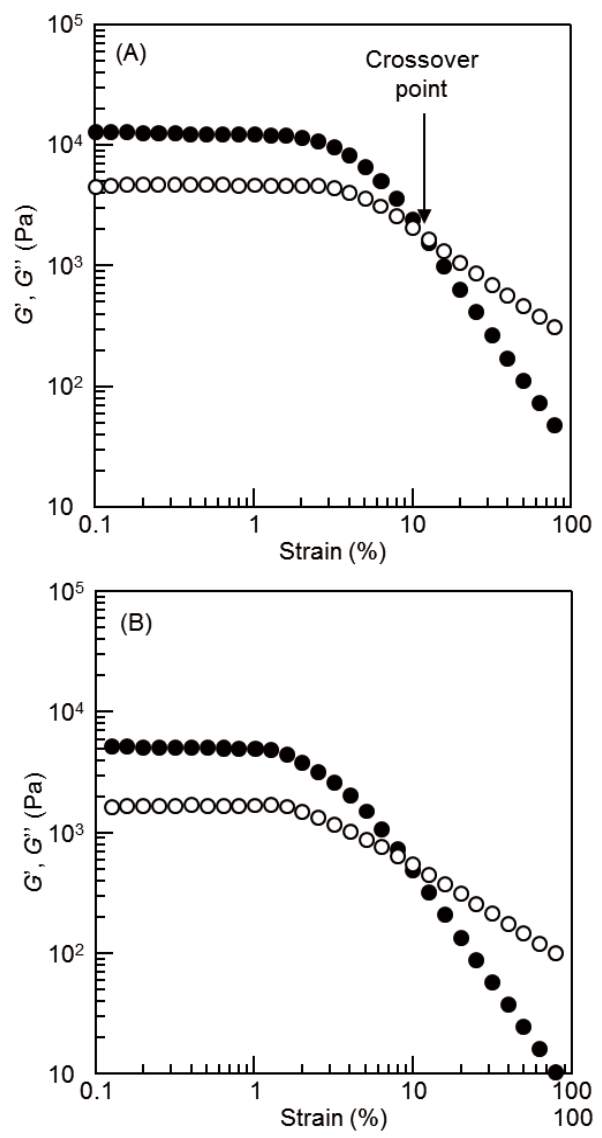

Fig. 5 Strain sweep tests of storage $\left(G^{\prime}, \bullet\right)$ and loss $\left(G^{\prime \prime}, \circ\right)$ moduli of particle/liquid-crystal composite gels with different $e$. e. values: (A) $0 \%$ (nematic phase); (B) $100 \%$ (cholesteric phase, pitch $/ 2=0.88 \mu \mathrm{m})$. The concentration of the chiral dopants was $5 \mathrm{wt} \%$ for the both composite gels. Experiments were conducted at $25{ }^{\circ} \mathrm{C}$ and a constant frequency of oscillation $(1 \mathrm{~Hz})$. 


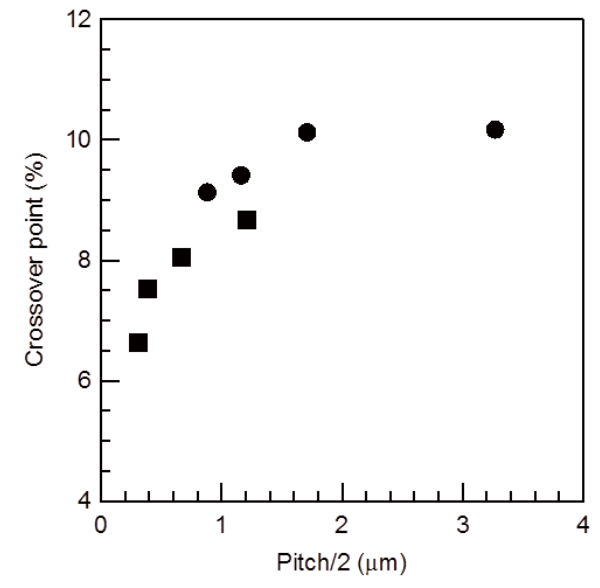

Fig. 6 Plots of crossover points of storage and loss moduli particle/LC composite gels as a function of pitch $/ 2$ values of cholesteric LCs. The concentrations of dopant mixture were 5 $(\bullet)$ and $15(\mathbf{a}) \mathrm{wt} \%$. Experiments were carried out at $25{ }^{\circ} \mathrm{C}$.

network structures of particles would be difficult in cholesteric LCs. In addition, the authors pointed out two important features: (i) the size of the two-dimensional plate clusters in cholesteric LCs became remarkably larger at the point where the pitch of a helical structure was significantly smaller than the particle size and (ii) a particle-particle distance slightly grew as the pitch of a helical structure became shorter. From these features, we qualitatively speculate here the origins of the softening of the particle/LC composite gels induced by the helical structures. By the introduction of the helical structure into a host LC, particles could not form networks efficiently due to the predominant formation of two-dimensional plate clusters. As a result, the composite gel became soft. Then, as the pitch of a helical structure became shorter with increasing the $e$.e. value, the composite gel exhibited a further softening. This would be due to the increase of a particle-particle distance. Furthermore, as the pitch of a helical structure was shorter than the particle size, the networks became fragile. The reduction of the toughness of the composite gels would be caused by a significant enhancement of the formation of two-dimensional plates in host LCs.

\section{CONCLUSIONS}

In this study, we found that the phase structures (nematic and cholesteric phases) of a host matrix had significant effects on the viscoelastic properties of particle/LC composite gels. The composite gel became soft by the introduction of a helical structure into a host LC. Storage modulus indicating the rigidness of the composite gels decreased as pitch/2 values of a helical structure became smaller. Crossover points which denoted the toughness of the composite gels exhibited no significant changes when the pitch $/ 2$ values were larger than approximately $1.8 \mu \mathrm{m}$ which was coincided with the radius of particle used in this study. When the $\mathrm{pitch} / 2$ values were smaller than $1.8 \mu \mathrm{m}$, the toughness of the composite gels was significantly decreased. The differences in self-organization behavior of particles in nematic and cholesteric LCs will strongly affect the network formation of particles in host LCs and the viscoelastic properties of particle/LC composite gels.

References

[1] S. P. Meeker, W. C. K. Poon, J. Crain, and E. M. Terentjev, Phys. Rev. E, 61, R6083-R6086 (2000).

[2] V. J. Anderson, E. M. Terentjev, S. P. Meeker, J. Crain, and W. C. K. Poon, Eur. Phys. J. E, 4, 11-20 (2001)

[3] V. J. Anderson and E. M. Terentjev, Eur. Phys. J. E, 4, 21-28 (2001).

[4] P. G. Petrov and E. M. Terentjev, Langmuir, 17, 2942-2949 (2001).

[5] D. Vollmer, G. Hinze, B. Ullrich, W. C. K. Poon, M. E. Cates, and A. B. Schofield, Langmuir, 21, 4921-4930 (2005).

[6] N. Hijnen, T. A. Wood, D. Wilson, and P. S. Clegg, Langmuir, 26, 13502-13510 (2010).

[7] M. Roth, M. D'Acunzi, D. Vollmer, and G. K. Auernhammer, J. Chem. Phys., 132, 124702 (2010).

[8] T. A. Wood, J. S. Lintuvuori, A. B. Schofield, D. Marenduzzo, and W. C. K. Poon, Science, 333, 79-83 (2011).

[9] T. Yamamoto and M. Yoshida, Langmuir, 28, 8463-8469 (2012).

[10] T. Yamamoto, Y. Kawata, and M. Yoshida, $J$ Colloid Interf Sci, 397, 131-136 (2013).

[11] A. Agarwal, E. Huang, S. Palecek, and N. L. Abbott, Adv. Mater., 20, 4804-4809 (2008).

[12] S. K. Pal, A. Agarwal, and N. L. Abbott, Small, 5, 2589-2596 (2009).

(Received January 27, 2015; Accepted April 27, 2015) 